\title{
How Can Artificial Intelligence Make Medicine More Preemptive?
}

Usman Iqbal $^{1,2}$, PharmD, MBA, PhD; Leo Anthony Celi ${ }^{3,4,5}$, MD, MSc; Yu-Chuan Jack $\mathrm{Li}^{2,6,7,8}$, MD, PhD

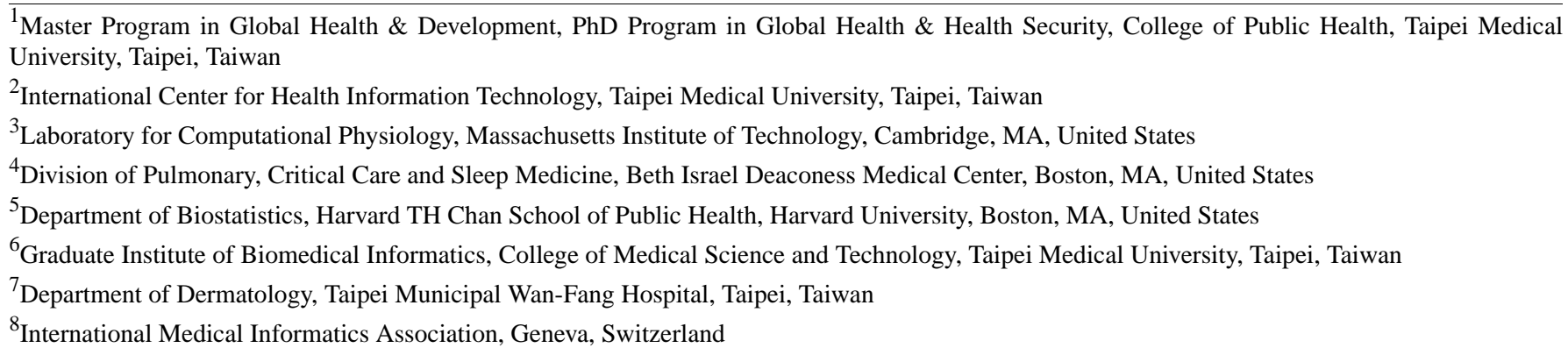

Corresponding Author:

Yu-Chuan Jack Li, MD, PhD

Graduate Institute of Biomedical Informatics

College of Medical Science and Technology

Taipei Medical University

No 172-1, Sec 2, Keelung Rd

Daan District

Taipei, 10675

Taiwan

Phone: 88666382736 ext 7601

Email: jack@tmu.edu.tw

\section{Related Article:}

This is a corrected version. See correction statement in: http://www.jmir.org/2020/8/e23645/

\begin{abstract}
In this paper we propose the idea that Artificial intelligence (AI) is ushering in a new era of "Earlier Medicine," which is a predictive approach for disease prevention based on AI modeling and big data. The flourishing health care technological landscape is showing great potential-from diagnosis and prescription automation to the early detection of disease through efficient and cost-effective patient data screening tools that benefit from the predictive capabilities of AI. Monitoring the trajectories of both in- and outpatients has proven to be a task AI can perform to a reliable degree. Predictions can be a significant advantage to health care if they are accurate, prompt, and can be personalized and acted upon efficiently. This is where AI plays a crucial role in "Earlier Medicine" implementation.
\end{abstract}

(J Med Internet Res 2020;22(8):e17211) doi: $\underline{10.2196 / 17211}$

\section{KEYWORDS}

artificial intelligence; digital health; eHealth; health care technology; medical innovations; health information technology; advanced care systems

In this paper we propose the idea that artificial intelligence (AI) is ushering in a new era of "Earlier Medicine." Advanced digital health solutions play a significant role in improving health care by enabling accurate diagnosis, prescription automation, and early prediction of diseases with potential AI capabilities [1,2]. "Earlier Medicine" refers to a temporally predictive and proactive approach to individualized health enabled by innovative AI modeling plus longitudinal/personal health big data. It calls on medical practice to not just react or manage present situations but also medical events of the foreseeable future. Such an approach will save money, lives, and our health care ecosystem from inefficiencies and disintegration.

Monitoring the trajectories of both in- and outpatients has proven to be a task AI can perform reliably [3]. Predictive tools present a considerable advantage to medicine if they are accurate, prompt, customizable, and actionable. This is where AI plays a crucial role in "Earlier Medicine" implementation. In the late 1940s, Leavell and Clark [4] proposed three levels of prevention: primary, secondary, and tertiary. The term "primary prevention" 
was used to describe "measures applicable to a particular disease or group of diseases to intercept the causes of disease before they involve man" [4]. It is important to note, however, that the concept of prevention has evolved with time. Leavell and Clark's definition is disease-oriented, but the application of prevention overall extends beyond medical problems and addresses other societal concerns. Through prevention, we can create a society that fosters good health to improve the quality of care [5].

In the 1970s, Schwartz [6] speculated that by the year 2000, a sizeable amount of the thought process involved in medicine could be augmented by a division of AI known as expert systems. Recently, Topol [7] proposed that new technologies will improve the precision and accuracy of diagnosis and in doing so will enhance treatment selection. In the last 2 years, the World Health Organization (WHO) has been reviewing evidence on digital technologies from consulting experts around the world in order to assemble recommendations on ways such tools can be used for maximum impact on health care. In 2018, governments unanimously adopted a World Health Assembly resolution, calling on the WHO to develop a global digital health strategy to support national efforts to achieve universal health coverage [8]. The United States Food and Drug Administration has been proposing ways to establish greater oversight over this rapidly evolving segment of AI products to regulate these systems whose performance constantly change based on exposure to new patient data in different clinical settings [9]. Keeping these initiatives in mind, the saying, "prevention is better than cure" can be realized in a way like never before due to the advent of AI for "Earlier Medicine." This brings us to propose the following levels of prevention-Actionable, Accurate, Timely, and Individualized (Figure 1).

Figure 1. Artificial intelligence (AI) for "Earlier Medicine" with primary, secondary, and tertiary prevention levels.

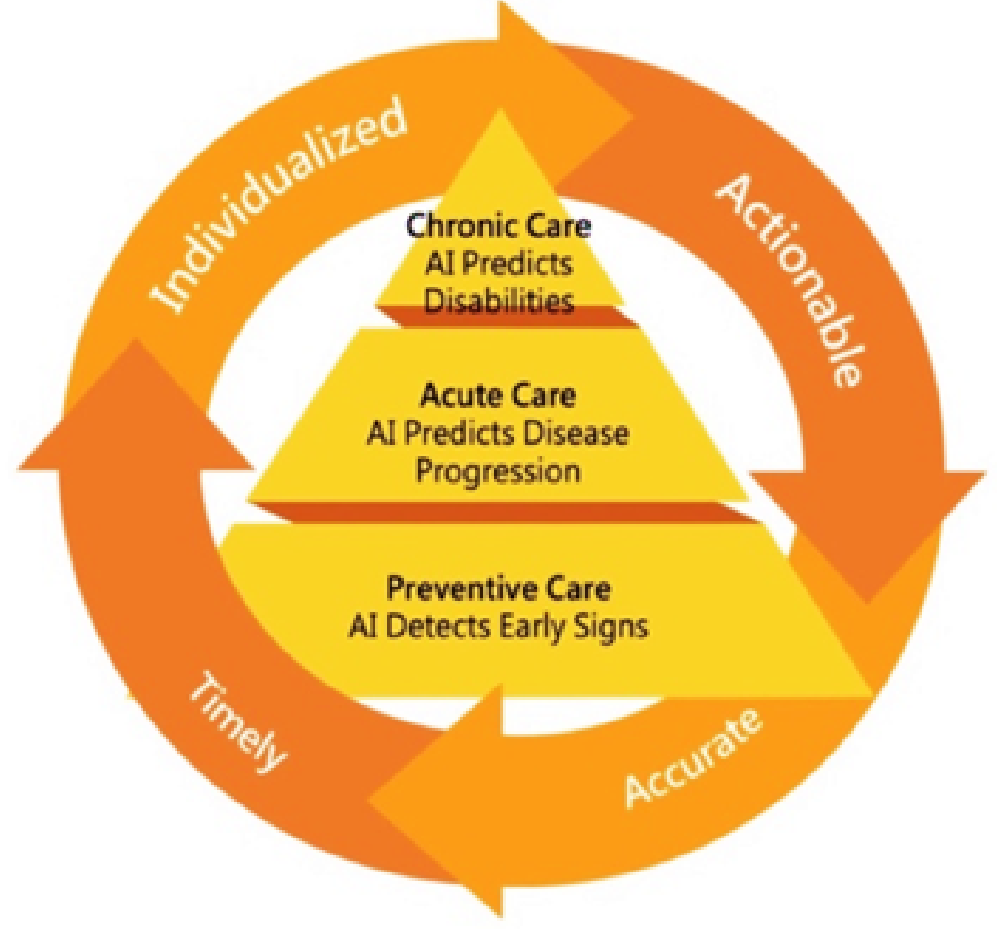

First, we propose that primary prevention using AI should be targeted at those who are well. In the case of screening all healthy individuals for risk of disease, AI can be used for earlier rather than early detection for risk reduction. However, current screening protocols are oversimplified and suffer from low compliance. More robust models are needed, for example, for the risk prediction for nonmelanoma skin cancer [10]. Screening procedures are often the first step leading to early interventions that are more cost-effective than intervening once symptoms appear. In United States, positive results from screening mammograms were of little benefit; they resulted in increases to costs, and did little to nothing to ensure quality of life and decrease mortality rate [11]. Early detection serves little purpose for patients with an illness. We believe that society would benefit more from increased precision in the selection of groups for screening with AI-based earlier risk reduction using AI prediction technology. Other examples of the possible use of AI prediction technology is in the diagnosis of some forms of melanoma from an atypical mole such as cutaneous pigmented lesion screening, using smartphone-generated images and clinical information simultaneously [12]. The incorporation of genomic information in electronic health records as part of one's personalized treatment will drive the greater use of AI in primary prevention [13].

Secondly, we advocate that "Earlier Medicine" for secondary prevention is just as crucial. For a patient who is at risk of or suffering from a disease, AI can compute a management plan tailored to the patient's individual needs. Secondary prevention AI includes recurrence prediction (eg, predicting non-ST-elevation myocardial infarction for patients with chest pain [14]).

Third, "Earlier Medicine" for tertiary prevention focuses on the deterrence of consequences of disease such as complications and disability. It also focuses on the overall improvement of quality of life through AI-based earlier interventions. Most 
countries are experiencing increases in the proportion of older people that comprise their population. It is estimated that by 2030 , around $22 \%$ of the world's population will be over 60 years of age [15]. There is an increasing prevalence of chronic disease in this age bracket globally and assisted daily living levels vary among regions $[16,17]$. Current health care systems that deal with this leave much to be desired in terms of long-term care, palliative care, and the expenses needed to maintain the system. Once a developed disease has been treated during its acute clinical phase, tertiary prevention seeks to soften the impact caused by the disease and potential complications on the patient's function, longevity, and quality of life. Tertiary prevention can include modifying risk factors. In cases where the condition is not reversible, tertiary prevention focuses on rehabilitation, assisting the patient to accommodate to disability [18]. For example, "Earlier Medicine" for tertiary prevention based on AI-computed individualized risk would help to prevent falls at home by $90 \%$ while reducing at least $10 \%$ of the dependent cost [19]. The key goal of tertiary prevention is to enhance quality of life by focusing on home health care services for either a short or long period as a result of illness, impaired health, old age, or other factors [20,21].

We earnestly believe that AI for "Earlier Medicine" will not only transform the practice of medicine but also radically reshape health care around the world. Harnessing the power of digital technologies is essential for achieving universal health coverage and to reviving humane medical practices for improving the quality of care [22]. The idea of prevention has evolved over the years from primary to tertiary and from a doctor-driven to patient-centered care model. AI for "Earlier Medicine" can serve as virtual medical assistants for clinicians, allowing for the resurgence of empathy-based care [23].

\section{Acknowledgments}

LAC is funded by the National Institutes of Health through NIBIB R01 EB017205.

\section{Conflicts of Interest}

None declared.

\section{References}

1. Ahuja AS. The impact of artificial intelligence in medicine on the future role of the physician. PeerJ 2019 Oct;7:e7702 [FREE Full text] [doi: 10.7717/peerj.7702] [Medline: 31592346]

2. Kukafka R. Digital Health Consumers on the Road to the Future. J Med Internet Res 2019 Nov 21;21(11):e16359 [FREE Full text] [doi: 10.2196/16359] [Medline: 31750835$]$

3. Parreco J, Hidalgo A, Parks JJ, Kozol R, Rattan R. Using artificial intelligence to predict prolonged mechanical ventilation and tracheostomy placement. J Surg Res 2018 Aug;228:179-187. [doi: 10.1016/j.jss.2018.03.028] [Medline: 29907209]

4. Top FH. Preventive Medicine for the Doctor in His Community: An Epidemiologic Approach. Arch Intern Med 1959 Jan 01;103(1):164. [doi: 10.1001/archinte.1959.00270010170027]

5. Glossary of terms used in the "Health for All" series, no. 1-8. World Health Organization. 1984. URL: https://apps.who.int/ iris/handle/10665/39565 [accessed 2020-07-02]

6. Schwartz WB. Medicine and the Computer. N Engl J Med 1970 Dec 03;283(23):1257-1264. [doi: 10.1056/nejm197012032832305]

7. Topol E. Deep Medicine: How Artificial Intelligence Can Make Healthcare Human Again. UK: Hachette; Mar $12,2019$.

8. Seventy-first World Health Assembly update, 25 May. World Health Organization. 2018 May 25. URL: https://www. who.int/news-room/detail/25-05-2018-seventy-first-world-health-assembly-update-25-may [accessed 2020-07-02]

9. Proposed regulatory framework for modifications to artificial intelligence/machine learning (AI/ML)-based software as a medical device (SAMD): discussion paper and request for feedback. United States Food and Drug Administration. 2019. URL: https://www.fda.gov/files/medical\%20devices/published/ US-FDA-Artificial-Intelligence-and-Machine-Learning-Discussion-Paper.pdf [accessed 2020-07-02]

10. Wang H, Wang Y, Liang C, Li Y. Assessment of Deep Learning Using Nonimaging Information and Sequential Medical Records to Develop a Prediction Model for Nonmelanoma Skin Cancer. JAMA Dermatol 2019 Sep 04;155(11):1277. [doi: 10.1001/jamadermatol.2019.2335] [Medline: 31483437]

11. Morris E, Feig SA, Drexler M, Lehman C. Implications of Overdiagnosis: Impact on Screening Mammography Practices. Popul Health Manag 2015 Sep;18 Suppl 1(S1):S3-11. [doi: 10.1089/pop.2015.29023.mor] [Medline: 26414384]

12. Chin Y, Hou ZY, Lee MY, Chu HM, Wang HH, Lin YT, et al. A patient-oriented, general-practitioner-level, deep-learning-based cutaneous pigmented lesion risk classifier on a smartphone. Br J Dermatol 2020 Jun 01;182(6):1498-1500. [doi: 10.1111/bjd.18859] [Medline: $\underline{31907926]}$

13. Hazin R, Brothers KB, Malin BA, Koenig BA, Sanderson SC, Rothstein MA, et al. Ethical, legal, and social implications of incorporating genomic information into electronic health records. Genet Med 2013 Oct 12;15(10):810-816 [FREE Full text] [doi: 10.1038/gim.2013.117] [Medline: 24030434]

14. Wu C, Hsu W, Islam MM, Poly TN, Yang H, Nguyen P, et al. An artificial intelligence approach to early predict non-ST-elevation myocardial infarction patients with chest pain. Comput Methods Programs Biomed 2019 May;173:109-117. [doi: 10.1016/j.cmpb.2019.01.013] [Medline: $\underline{\text { 31046985] }}$ 
15. Department of Economic and Social Affairs, Population Division. World Population Ageing 2019. New York, NY: United Nations; 2019. URL: https://www.un.org/en/development/desa/population/publications/pdf/ageing/

WorldPopulationAgeing2019-Highlights.pdf [accessed 2020-07-02]

16. Scheel-Hincke LL, Möller S, Lindahl-Jacobsen R, Jeune B, Ahrenfeldt LJ. Cross-national comparison of sex differences in ADL and IADL in Europe: findings from SHARE. Eur J Ageing 2020 Mar 14;17(1):69-79. [doi: 10.1007/s10433-019-00524-y] [Medline: 32158373]

17. Kuo H, Lin K, Lan C, Li I. Activities of daily living trajectories among institutionalised older adults: A prospective study. J Clin Nurs 2017 Dec 23;26(23-24):4756-4767. [doi: 10.1111/jocn.13828] [Medline: 28334483]

18. Cattelani L, Palumbo P, Palmerini L, Bandinelli S, Becker C, Chesani F, et al. FRAT-up, a Web-based fall-risk assessment tool for elderly people living in the community. J Med Internet Res 2015 Feb 18;17(2):e41 [FREE Full text] [doi: 10.2196/jmir.4064] [Medline: 25693419]

19. Gomez F, Curcio CL, Brennan-Olsen SL, Boersma D, Phu S, Vogrin S, et al. Effects of the falls and fractures clinic as an integrated multidisciplinary model of care in Australia: a pre-post study. BMJ Open 2019 Jul 29;9(7):e027013 [FREE Full text] [doi: 10.1136/bmjopen-2018-027013] [Medline: 31362962]

20. Kang H, Han J, Kwon GH. An Ecological Approach to Smart Homes for Health Care Services: Conceptual Framework of a Smart Servicescape Wheel. JMIR Mhealth Uhealth 2019 Feb 08;7(2):e12425 [FREE Full text] [doi: 10.2196/12425]

[Medline: $\underline{30735145]}$

21. Willis M, Duckworth P, Coulter A, Meyer ET, Osborne M. The Future of Health Care: Protocol for Measuring the Potential of Task Automation Grounded in the National Health Service Primary Care System. JMIR Res Protoc 2019 Apr 09;8(4):e11232 [FREE Full text] [doi: 10.2196/11232] [Medline: 30964437]

22. Rajkomar A, Dean J, Kohane I. Machine Learning in Medicine. N Engl J Med 2019 Apr 04;380(14):1347-1358. [doi: 10.1056/nejmra1814259]

23. Shen J, Zhang CJP, Jiang B, Chen J, Song J, Liu Z, et al. Artificial Intelligence Versus Clinicians in Disease Diagnosis: Systematic Review. JMIR Med Inform 2019 Aug 16;7(3):e10010 [FREE Full text] [doi: 10.2196/10010] [Medline: 31420959]

\section{Abbreviations}

AI: artificial intelligence

WHO: World Health Organization

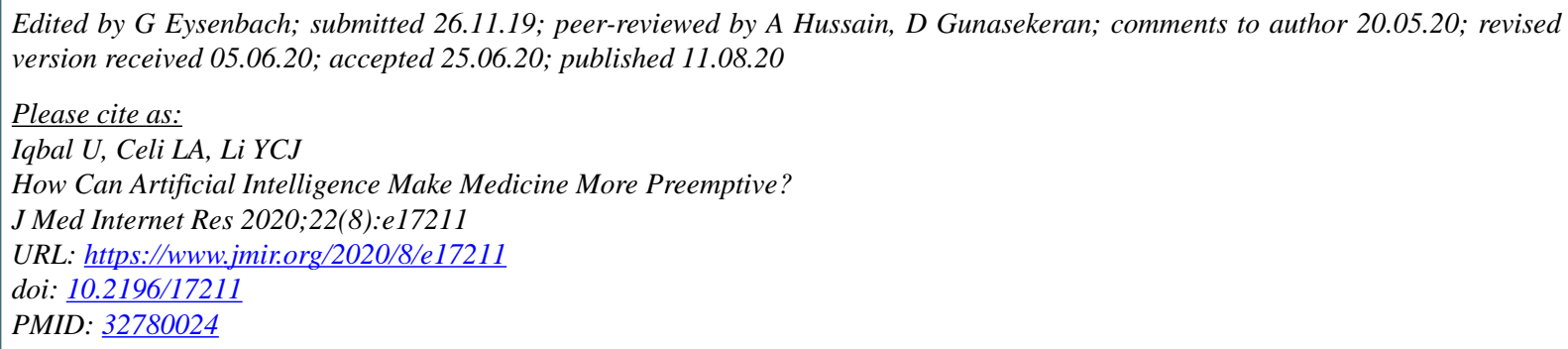

(CUsman Iqbal, Leo Anthony Celi, Yu-Chuan Jack Li. Originally published in the Journal of Medical Internet Research (http://www.jmir.org), 11.08.2020. This is an open-access article distributed under the terms of the Creative Commons Attribution License (https://creativecommons.org/licenses/by/4.0/), which permits unrestricted use, distribution, and reproduction in any medium, provided the original work, first published in the Journal of Medical Internet Research, is properly cited. The complete bibliographic information, a link to the original publication on http://www.jmir.org/, as well as this copyright and license information must be included. 